REGARDS

SUR LEECONOMIE ALLEMANDE

BULLETIN ECONOMIQUE DU CRAC

\section{Regards sur l'économie allemande}

Bulletin économique du CIRAC

$93 \mid 2009$

Varia

\title{
Mediencluster NRW : genèse d'un pôle de compétitivité
}

Isabelle Bourgeois et Werner Schwaderlapp

Traducteur : Isabelle Bourgeois

\section{OpenEdition}

Journals

Édition électronique

URL : http://journals.openedition.org/rea/3893

DOI : 10.4000/rea.3893

ISBN : 978-2-8218-0882-9

ISSN : 1965-0787

Éditeur

CIRAC

Édition imprimée

Date de publication : 1 octobre 2009

Pagination : 16-22

ISSN : 1156-8992

\section{Référence électronique}

Isabelle Bourgeois et Werner Schwaderlapp, « Mediencluster NRW : genèse d'un pôle de compétitivité », Regards sur l'économie allemande [En ligne], 93 | octobre 2009, mis en ligne le 01 octobre 2011, consulté le 05 mai 2019. URL : http://journals.openedition.org/rea/3893 ; DOI : 10.4000/rea.3893 


\title{
Mediencluster NRW : genèse d'un pôle de compétitivité
}

\author{
Un entretien avec Werner Schwaderlapp
}

Le Prof. Werner Schwaderlapp est directeur du Medien+Entertainment Management Institut (me:mi) a qui a été confiée l'évaluation du potentiel médias de la NRW en prévision de la création du Mediencluster NRW. Il est membre du Conseil d'orientation chargé du suivi du cluster (Medienbeirat).

II s'agit de rester le site médiatique numéro $1 \ldots$

... et de pérenniser cet atout dans un contexte évolutif
Le gouvernement du Land de Rhénanie du Nord-Westphalie (NRW) a décidé au début de 2007, dans le cadre de sa politique d'innovation, de lancer un cluster médias (Mediencluster NRW ; voir supra). D'habitude, la politique des pôles de compétitivité concerne les technologies high-tech, comme les nano- ou les biotechnologies. Comment s'explique le choix du secteur des médias?

Depuis le début 2007, les fonds de soutien de l'UE peuvent aussi être mis en œuvre pour développer les atouts des régions concernées. Le gouvernement du Land a ainsi lancé le soutien de clusters dans 16 de ces domaines qui présentent à la fois un grand dynamisme et un fort potentiel dans l'économie de NRW : l'automobile ou les matières synthétiques - mais aussi les médias.

Mais pourquoi créer un cluster alors que la NRW est déjà le site médiatique par excellence de l'Allemagne?

Dans le domaine des médias, la Rhénanie du Nord-Westphalie doit affronter la concurrence de Berlin, Munich et Hambourg. Et dans le domaine des nouvelles activités comme les médias en ligne ou les jeux électroniques, elle rivalise avec d'autres sites encore, comme Francfort et Stuttgart. II s'agit donc pour le Land de consolider son avantage compétitif et de le développer dans les nouveaux segments de la branche des médias.

Ce cluster s'étend à l'ensemble du Land?

Oui, en principe, il s'étend à l'ensemble du territoire. Mais en réalité, il accorde la prépondérance à la région autour de Cologne, puisque c'est là que se concentrent pour l'essentiel les activités médiatiques du Land. La priorité accordée à cette région répond ainsi à l'approche de la théorie des clusters selon laquelle une économie accroît sa compétitivité lorsqu'elle oriente sa politique sur le développement de ses atouts et que, au lieu de pratiquer un soutien indifférencié à l'échelle de l'ensemble du territoire, elle axe son soutien non seulement sur les activités à fort potentiel mais aussi sur le territoire où se concentrent ces activités. Les villes du Moyen-Age nous fournissent un excellent exemple de cette approche, avec leur rue des bouchers ou leur rue des orfèvres.

Comment ce cluster s'insère-t-il dans la politique structurelle régionale du gouvernement Rüttgers (CDU/FDP) au pouvoir depuis 2005 ? Et en quoi cette politique se distingue-t-elle de celle des gouvernements précédents (SPD/Verts) ?

Les prédécesseurs de Jürgen Rüttgers (CDU) : les ministres-présidents Johannes Rau et Wolfgang Clement (tous deux SPD) ont toujours mené une politique des médias très active ; Wolfgang Clement même alors qu'il était seulement ministre de l'Economie et chef de la Chancellerie du Land sous Johannes Rau. L'approche de cette politique était déjà celle d'une politique structurelle ou économique! Et elle avait pour point de départ le lancement d'un secteur privé de la télévision en Allemagne. Celui-ci a fortement dynamisé le marché des médias, ne serait-ce qu'en termes de chiffre d'affaires publicitaire, dont la croissance s'établissait à 4 milliards $€$ par an. La moitié environ de ce montant allait à la Rhénanie du Nord-Westphalie qui avait su attirer sur son territoire la chaîne RTL, ce qui avait entraîné à sa suite la constitution d'un pôle d'activités liées.

La politique du gouvernement Rüttgers suit donc deux axes : pérenniser cet atout et l'adapter aux mutations du secteur générées par la diffusion d'Internet. Pour 
enclencher une nouvelle phase de croissance du marché des médias (essor des médias en ligne et de la convergence) et de l'économie du Land, il a donc mis à disposition 10 millions $€$ via un appel à projets, lancé en août 2008 et qui s'est traduit par la sélection de 12 projets au début de l'année [voir dans ce numéro ; IB]. II a ainsi mis en œuvre les recommandations que nous avions formulées dans notre rapport, à savoir miser sur le potentiel du site médiatique pour le hisser au rang de leader allemand (voire européen) dans l'économie numérique.

Sur deux points, le Mediencluster NRW semble se différencier des pratiques généralement en vigueur outre-Rhin : contrairement à la logique du bottom up qui caractérise la plupart des pôles de compétitivité, il semble largement piloté par le gouvernement; cela est d'autant plus surprenant que les médias sont un secteur qui échappe à l'exécutif de par la Constitution. Comment expliquer cela ?

Lors de l'ouverture de l'audiovisuel aux opérateurs privés dans les années 1980, les préoccupations du Land en matière de politique économique étaient largement guidées par les questions de régulation - en particulier celles liées à la répartition des fréquences terrestres. Celles-ci étaient alors (techniquement) déterminantes pour l'évolution du marché, contrairement à aujourd'hui, où elles jouent un rôle résiduel puisque $95 \%$ des foyers allemands reçoivent la télévision par câble ou satellite. Or cette situation appelait une réponse centrale, c'est-à-dire au niveau du gouvernement du Land qui avait à gérer le plan de fréquences en coopération avec les autres Länder.

Aujourd'hui, la donne est totalement différente. La principale caractéristique du marché des médias en ligne est son ubiquité ; sa régulation comme sa localisation échappent donc par définition à l'acteur politique régional. Certes, ce marché se développe dans une large mesure en complémentarité avec les activités des médias traditionnels, mais comme il est fondé sur l'immatériel, rien ne s'oppose à la mobilité de ses acteurs ni à leur migration d'un Land vers l'autre. Le cluster médias de la Rhénanie du Nord-Westphalie poursuit donc l'objectif de relier en réseau les acteurs de la branche présents dans son Land en apportant un soutien à leur coopération en partenariat, et de préserver ce faisant l'attractivité de son site médiatique, donc de limiter à terme les tentations migratoires.

En Rhénanie du Nord-Westphalie, Andreas Krautscheid cumule les fonctions de ministre chargé des relations avec le Bund, de ministre des Affaires européennes et de ministre des Médias. Une telle concentration des pouvoirs semble peu usuelle dans le paysage allemand...

... dans tous les Länder, ce sont les ministres-présidents, et donc la chancellerie, qui sont compétents pour les médias. En Rhénanie du Nord-Westphalie, il se trouve que les relations avec le Bund et les Affaires européennes - d'importance stratégique en politique - relèvent elles aussi du sommet de la hiérarchie, et que ces questions sont concentrées dans un ministère. Ce n'est probablement pas le fruit du hasard, puisqu'Andreas Krautscheid, qui était auparavant secrétaire d'Etat à la chancellerie, y exerçait les fonctions de porte-parole du gouvernement et que le marché des médias entrait également dans ses prérogatives. Et d'ailleurs, dans un précédent gouvernement, une secrétaire d'Etat, Miriam Meckel, a cumulé elle aussi pendant quelque temps les responsabilités en matière de médias et d'affaires européennes. Cela dit, l'idée de soutenir l'émergence d'un pôle médias (qui n'a été rebaptisé cluster que bien plus tard) est antérieure au cumul de ces fonctions. Et même aujourd'hui, cette concentration des pouvoirs reste toute relative, puisque le ministère des Affaires européennes (et des Médias) ne dispose pas seul de l'usage des fonds structurels européens ; la responsabilité principale en incombe à son homologue de l'Economie et, en dernier ressort, au ministreprésident..

Le ministre Krautscheid cherche donc à développer la compétitivité de son Land dans l'économie du savoir. Quel intérêt présentent les médias (de l'information au show biz), pour l'innovation et la compétitivité globale d'un Land?

Autrefois, c'est sur l'industrialisation que reposait l'essor de l'économie, puis, au début de l'ère de la société du savoir, sur la numérisation. Aujourd'hui, c'est aux
La priorité : réagir à la mobilité des acteurs en leur offrant un environnement attractif

Une concentration des pouvoirs toute relative

Un Land sans richesses naturelles doit miser sur l'intelligence 
La mission de l'institut me:mi : formuler des recommandations
Miser sur la formation continue le temps de développer les cursus de formation initiale appropriés industries culturelles et créatives qu'on attribue à plusieurs égards un rôle moteur dans la croissance macro-économique. C'est là notamment une thèse que défend l'économiste américain Richard Florida et que s'est appropriée le ministreprésident Rüttgers pour fonder sa politique de développement structurel. Elle entre également dans les réflexions du gouvernement fédéral qui a récemment publié un important rapport sur les industries culturelles et créatives. L'idée sur laquelle repose cette approche est simple : la prospérité d'un pays comme l'Allemagne qui ne dispose que de peu de ressources naturelles ne peut être portée que par une autre richesse - l'intelligence. C'est là qu'interviennent les industries dites créatives : d'une part, elles sont un élément de cette richesse ; d'autre part, elles créent un environnement stimulant pour les entreprises comme pour les Hommes, où ces derniers aiment travailler et qui les rend performants, et où les produits et services développés, largement immatériels, sont innovants.

\section{L'exemple type d'une politique d'offre}

Comment a-t-il été procédé concrètement pour la mise en œuvre du cluster médias ? Et quel a été le rôle de votre institut?

Après que le gouvernement a décidé que l'un des 16 clusters lancés dans le cadre de sa politique structurelle serait consacré à la promotion des médias, il a confié à l'institut me:mi la mission de réaliser un rapport pour dresser un état des lieux et formuler des recommandations d'action. Nous avons ainsi conseillé au gouvernement de centrer la promotion des médias sur la convergence à l'ère du numérique. Cela vise notamment le développement de nouveaux modèles économiques adaptés et porteurs. Car tout reste à inventer dans ce domaine. Ce n'était pas le cas au moment du lancement de la télévision privée dont le modèle économique était mondialement connu et assimilé en Allemagne : je recrute des téléspectateurs en leur proposant des émissions attractives, et je revends ces mêmes téléspectateurs aux annonceurs en tant que contacts publicitaires.

\section{Le Medien+Entertainment Management Institut (me:mi), Cologne}

La Hochschule Fresenius est une école supérieure spécialisée (Fachhochschule) privée créée voici plus de 160 ans par le chimiste du même nom pour former des chimistes. Aujourd'hui, elle dispense sur son site de Cologne des formations dans les filières sciences de gestion, économie des médias, économie de la santé, droit des affaires et psychologie économique (www.hsfresenius.de). Elle a ouvert en 2008 deux masters, dont un dénommé « Media Management \& Entrepreneurship ».

Elle abrite, à Cologne, un institut de recherche indépendant: le memi-Institut (www.memikoeln.de). Ce type de rapprochement (très répandu en Allemagne et connu sous la dénomination de An-Institut : " institut placé auprès de »; c'est par exemple le statut de l'institut de recherche économique de Kiel, l'IfW) signifie que si, juridiquement, l'institut est indépendant de l'université, un certain nombre de liens l'associent néanmoins à l'université. Ils se manifestent en particulier par des missions de recherche et le développement de projets, ainsi que la coopération au niveau des enseignements.

L'institut me:mi, créé en 2004, exerce une double mission: recherche appliquée au monde professionnel en amont ; en aval, conseil aux entreprises et institutions du secteur des médias et de l'entertainment.

Le Prof. Werner Schwaderlapp, fondateur et directeur de l'institut me:mi, est également cofondateur et coactionnaire de l'antenne de Cologne de la Hochschule Fresenius. [IB]

Vos recommandations portent principalement sur deux points : « formation continue convergente " et " entreprenariat ". Pourriez-vous nous en dire plus ?

En Allemagne, et tout particulièrement dans l'agglomération de Cologne, nous avons une excellente offre de formation dans les médias traditionnels - du journalisme au cinéma. C'est sur la base de ces compétences acquises par les collaborateurs dans le secteur des médias qu'il convient, à notre avis de d'offrir dans un premier temps une formation continue adaptée à la convergence. Car la création d'une nouvelle offre de formation initiale prend en règle générale un à deux ans, et sa mise en œuvre dure au moins trois ans supplémentaires ; il faut donc compter au moins cinq ans avant de la voir porter ses premiers fruits. 
C'est un laps de temps beaucoup trop long pour un processus dynamique comme la convergence. Nous développerons aussi la formation initiale - mais plus tard.

Pour ce qui est de "l'entreprenariat ", il ne s'agit pas tant de diffuser un vent nouveau dans l'approche managériale des entreprises existantes (qu'il s'agisse de PME ou de grands groupes), mais bien plutôt d'impulser le développement d'approches réellement nouvelles - donc de contribuer à faire naître de nouvelles idées, des contenus innovants, de même que des modèles économiques porteurs. Pour cela, il faut qu'émerge une nouvelle culture d'entreprise : soit sous la forme de la création autonome d'une entreprise (le soutien vise alors l'entrepreneur) soit sous celle de la diffusion d'une culture innovante partagée par les salariés au sein de l'entreprise même (ce que nous appelons le soutien à « l'intrapreneur »).

A l'été 2008 ont été constitués un Conseil d'orientation appelé Medienbeirat, ainsi qu'un jury chargé de sélectionner les projets. Quelle est l'articulation entre ces deux institutions?

Le Medienbeirat s'est réuni deux fois jusqu'ici. Composé essentiellement de professionnels et de représentants des organisations de la branche, il a pour mission d'assurer une veille de l'évolution de la branche et, sur la base de ses observations et réflexions, de formuler des recommandations pour le développement ultérieur du cluster. Quant au jury, composé lui aussi de personnalités du 'métier', il est exclusivement chargé de sélectionner les projets à promouvoir.
Aider à l'émergence d'une culture d'entreprise nouvelle

Conseil d'orientation et jurys composés d'acteurs du métier

\begin{tabular}{|c|c|}
\hline \multicolumn{2}{|c|}{ Mediencluster NRW } \\
\hline Les membres du Medienbeirat... & ... et du jury \\
\hline \multicolumn{2}{|c|}{ Audiovisuel et cinéma : } \\
\hline $\begin{array}{l}\text { Verena Kulenkampff, } \\
\text { WDR (Cologne) } \\
\text { Michael Schmid-Ospach, } \\
\text { Filmstiftung NRW (Düsseldorf) } \\
\text { Simone Stewens, } \\
\text { Internationale Filmschule (Cologne) } \\
\text { Rafaela Wilde, } \\
\text { film \& fernsehproduzentenverband nrw (Cologne) } \\
\text { Sönke Wortmann, } \\
\text { Little Shark Entertainment (Cologne) }\end{array}$ & $\begin{array}{l}\text { Claude Schmit, } \\
\text { Super RTL (Cologne) } \\
\text { Prof. Katrin Schlösser, } \\
\text { Kunsthochschule für Medien (Cologne) }\end{array}$ \\
\hline \multicolumn{2}{|l|}{ Médias de l'écrit } \\
\hline $\begin{array}{l}\text { Katharina Borchert, Der Westen (Essen) } \\
\text { Konstantin Neven DuMont, Editions M. Dumont Schauberg (Cologne) }\end{array}$ & $\begin{array}{l}\text { Udo Becker, Bund Deutscher Zeitungsverleger } \\
\text { NRW (Düsseldorf) }\end{array}$ \\
\hline \multicolumn{2}{|c|}{ Télécommunications et médias électroniques/Internet } \\
\hline $\begin{array}{l}\text { Olaf Coenen, EA Electronic Arts (Cologne) } \\
\text { Thomas Ellerbeck, Vodafone (Düsseldorf) } \\
\text { Ibrahim Evsan, Sevenload (Cologne) } \\
\text { Florian Koch, BITKOM (Berlin) } \\
\text { Christopher Schläffer, Deutsche Telekom (Bonn) } \\
\text { Marc Schröder, RTL Interactive (Cologne) } \\
\text { Marco Zingler, Bundesverband Digitale Wirtschaft, et Denkwerk GmbH } \\
\text { (Cologne) }\end{array}$ & $\begin{array}{l}\text { Michael Loeb, WDR mediagroup (Cologne) } \\
\text { Harald A. Summa, Verband der deutschen } \\
\text { Internetwirtschaft (Cologne) } \\
\text { Christiane Siwek, Bundesverband Digitale } \\
\text { Wirtschaft (Düsseldorf) }\end{array}$ \\
\hline \multicolumn{2}{|c|}{ Expertise/formation/recherche } \\
\hline $\begin{array}{l}\text { Lutz Hachmeister, HMR International (Cologne) } \\
\text { Werner Schwaderlapp, Medien+Entertainment Management Institut } \\
\text { (Cologne) }\end{array}$ & $\begin{array}{l}\text { Prof. Jörg Müller-Lietzkow, Université de } \\
\text { Paderborn }\end{array}$ \\
\hline \multicolumn{2}{|l|}{ Autres } \\
\hline $\begin{array}{l}\text { Arno Füser, Fortis Bank SA/NV (Cologne) } \\
\text { Ralf Schremper, Bertelsmann (Gütersloh) }\end{array}$ & $\begin{array}{l}\text { Kai Röffen, TWWA et Art Directors Club } \\
\text { Deutschland (Düsseldorf) } \\
\text { Rainer Virnich, Sparkasse Cologne-Bonn }\end{array}$ \\
\hline
\end{tabular}


L'appel à projets ne fixait qu'un cadre d'orientation très général

Miser sur l'atout qu'est la présence de la GamesCom à Cologne

La créativité, l'innovation, ne peuvent naître que du marché...

... et leur produit doit s'auto-financer à terme

Un management professionnel pour le cluster
L'appel à projets était ainsi formulé: « En quête des meilleures idées pour l'innovation, la convergence et la mise en réseau des médias ". C'est très vague. Quels ont été les critères appliqués pour la sélection des 12 projets finalement retenus ?

Sous ce titre général, l'appel à projets développait une série de priorités thématiques, et fixait donc un cadre d'orientation général. Les projets retenus ont bien identifié les thématiques d'avenir : si on y regarde de près, la communication en ligne n'a rien à voir avec la communication de masse, puisqu'elle s'effectue d'individu à individu dans un processus interactif, même si ce processus touche les masses. II y a toujours à la base une liaison point-à-point, ce qui fait du consommateur des services en ligne le destinataire individuel du message. Cela ouvre de nouvelles perspectives en termes de contenus, de ciblage et donc aussi de modèles économiques. Les thématiques des projets retenus concernent donc la publicité ciblée, les services mobiles contextuels régionaux, les portails personnalisés d'informations régionales, et les jeux électroniques (technologies, contenus, formation).

Qu'est-ce qui a déterminé le choix de ces thématiques ? Et plus particulièrement, pourquoi les jeux électroniques?

Les thématiques évoquées ne s'entendent que comme quelques exemples de champs d'activités à fort potentiel économique. Parallèlement, l'appel à projet comprenait un volet "wild card », où toute autre proposition était la bienvenue. Pour ce qui est des jeux, ils font partie des médias d'avenir. Et s'ils jouissent d'une grande attention en Rhénanie du Nord-Westphalie, c'est que Cologne a réussi à s'imposer pour accueillir dorénavant le salon européen GamesCom (pour la première fois en août 2009) et à supplanter les sites auxquels on attribue d'habitude les plus grands attraits comme Barcelone, Londres ou encore Cannes.

Ce qui frappe, quand on considère la composition du Medienbeirat et du jury, c'est le rôle primordial dévolu aux acteurs de la branche (entreprises, fédérations)...

Le rôle de l'Etat n'est pas d'agir en entrepreneur. II consiste bien plutôt à faciliter et à soutenir l'activité des entreprises. C'est la pluralité des acteurs en présence, et même le chaos que peut présenter parfois un marché qui font jaillir l'innovation, jamais ou presque la mise en œuvre de directives émanant de la haute autorité de l'Etat. La sagesse, la créativité, ne sont pas des dons que Dieu aurait réservés aux monarques ou à leur successeurs démocratiquement élus, mais ils appartiennent à tous et à chacun, et leurs fruits sont omniprésents. Lorsque la nouvelle chaîne RTL s'était installée dans le Land, le gouvernement n'avait pas émis la moindre recommandation quant aux émissions qu'elle devait produire, aux chaînes qu'elle devait rassembler sous le toit de la "famille RTL ", ou sur la question de savoir si, après la création de RTL interactive, il convenait ou non d'étendre la marque à d'autres médias encore.

Et dans le cadre du cluster médias, les projets soutenus doivent apporter $50 \%$ du financement du projet, ce qui est en quelque sorte une garantie politique de sa viabilité, puisque cet apport financier prouve que le projet a été dûment évalué en ce qui concerne sa pertinence économique, c'est-à-dire qu'il est susceptible de trouver sa place sur le marché et, à terme, de générer des profits sans l'aide de subventions. C'est ainsi qu'on pourrait résumer l'approche en termes de politique d'offre qui préside en Allemagne à toute politique active de soutien à l'économie.

\section{Qui pilote le Mediencluster NRW ?}

C'est le ministre des Médias qui, par l'intermédiaire de la banque publique régionale du Land, la NRW.Bank, pilote le cluster pour l'instant, en attendant que soient institutionnalisés les fonctions décisionnelles et de conseil. Depuis juillet 2009, le management opérationnel du cluster est exercé par une filiale de la NRW.BANK ; elle a pour mission de gérer l'ensemble des fonds de soutien publics. Le directeur de cette société, la Mediencluster NRW GmbH (Cologne), a pris ses fonctions le 20 juillet. Marc Ziegler (40 ans) a été choisi pour son expérience professionnelle en la matière : il est conseiller pour les entreprises du domaine des médias, des télécommunications et de l'Internet. 
Comment s'est faite concrètement l'attribution des fonds aux projets retenus?

Pour le premier appel à projets, c'est la NRW.Bank qui a été chargée de la mise en oeuvre du financement pour le compte du gouvernement ; elle en a aussi géré l'administration, y compris sous l'angle juridique, conformément à sa mission d'administration déléguée. En ce qui concerne les thématiques des projets, la NRW.BANK s'est appuyée entre autres sur les conseils de l'institut me:mi qui était, pour sa part, chargé de formuler les orientations sous l'angle de l'économie du secteur des médias et d'en contrôler le suivi.

\section{Comment s'articule la coopération avec le Conseil d'orientation ?}

Le Medienbeirat recueille les avis sur les thématiques décidées et sur les résultats observés. Et il joue un rôle consultatif dans la gestion du cluster. Ces processus qui s'effectuaient souvent aussi de manière informelle jusqu'ici recevront une base plus institutionnelle avec l'organisation du management de ce cluster et la création de la société chargée de l'exercer.

\section{Seules les PME ont saisi « l'enjeu de la mise en concurrence des idées par appel à projets ॥}

L'un des objectifs poursuivis dans le cadre de ce cluster est le soutien aux PME, un axe politique d'ordinaire transversal. Comment a-t-il été décliné au niveau sectoriel?

Les chambres de commerce et d'industrie de Cologne, Düsseldorf et Dortmund ont organisé, lors du lancement de l'appel à projets, des séminaires d'information pour les entreprises intéressées. La chancellerie, la NRW.BANK et l'institut me:mi en ont à cette occasion explicité le contenu.

Quel rôle ont joué les 'géants' du Land : le groupe multimédias Bertelsmann, l’opérateur Deutsche Telekom, le groupe de presse WAZ, l'établissement audiovisuel public WDR ?

Ces grands groupes n'ont pas encore saisi entièrement l'enjeu de la mise en concurrence des idées par appel à projets. Dans l'intérêt de la bonne constitution du réseau, il serait souhaitable qu'ils s'impliquent plus lors du prochain. Car l'un des objectifs de ce programme est que des entreprises aux compétences clefs les plus diverses développent dans la région des projets conjoints. Les projets déposés en partenariat présentent en effet un certain nombre d'avantages sur ceux soumis par une entreprise isolée. Tous les projets présentés lors du premier appel étaient d'ailleurs établis sur la base du partenariat.

\section{Comment se présente la suite?}

Le $1^{\text {er }}$ octobre, le ministre des Médias a lancé un second appel à projets doté de 15 millions $€$. Théoriquement, le cluster media entre en concurrence avec les 15 autres clusters du Land pour l'attribution de ces fonds de soutien d'origine communautaire ou régionale. L'attribution de fonds européens présupposant un apport équivalent de la part du Land, il n'est pas possible actuellement de prévoir quel sera le montant total définitif des aides.

\section{Quelles sont les priorités thématiques de ce second appel à projets ?}

Idées et modèles économiques innovants. Plus concrètement, sont visés les contenus et modèles économiques dans le créneau des médias en ligne, les applications mobiles, et «la presse quotidienne du futur ». Dans ce cas aussi, est prévu une « wildcard », volet ouvert d'appel à idées.

Le lancement de ce Mediencluster NRW a finalement été très peu thématisé dans l'espace public. Et quand c'est le cas, la presse spécialisée tend plutôt à ironiser sur cette prédilection que semblent montrer pour les médias les gouvernements successifs du Land...

... le premier appel à projets a tout de même mobilisé 400 entreprises, les amenant à se pencher sur des préoccupations d'avenir. 125 entreprises se sont por-
Plusieurs critères et instances pour l'attribution des fonds

Les $\mathrm{CCl}$ ont ciblé les PME

Faible participation des grands groupes

Un $2^{\text {eme }}$ appel à projets lancé le $1^{\text {er }}$ octobre 2009

Mieux faire connaître le cluster dans l'espace public 
tées candidates, soit en tant que responsables d'un consortium, soit en tant que partenaires dans un tel réseau. Et le jury a finalement retenu 12 projets. Je n'ai pas connaissance d'un engagement comparable des acteurs de la branche dans aucun des autres sites médiatiques allemands. Cela dit, il est vrai que la chancellerie n'a peut-être, au goût de la branche, pas assez communiqué sur ce projet dans l'espace public, mais sa politique de communication s'adressait en priorité au monde professionnel.

Bien qu'il soit orienté sur les évolutions futures (forcément incertaines) d'une 'branche', l'objectif poursuivi ne s'en assimile pas moins à un soutien sectoriel...

... la stratégie que poursuit ce cluster médias est de renforcer la compétitivité du Land de Rhénanie du Nord-Westphalie face à la concurrence des autres sites médiatiques allemands - mais aussi de mieux le positionner sur l'échiquier européen. En ce sens, son objectif est celui d'une politique industrielle ciblée...

... au point de vue sectoriel, mais encore plus régional. A voir la localisation des projets soutenus, on serait tenté de rebaptiser le projet: "Mediencluster Cologne $» !$...

... ce qui correspondrait de fait à la réalité. Et il faut d'ailleurs qu'il en soit ainsi pour que le projet soit couronné de succès. Cela étant, jamais un gouvernement, que ce soit celui de la Rhénanie du Nord-Westphalie ou d'un autre Land, ne se risquerait bien sûr à affirmer que rien d'intéressant n'est susceptible de se produire dans d'autres régions. Mais une politique qui consisterait à répartir les fonds de soutien de manière indifférenciée sur l'ensemble du territoire serait contraire à l'approche même de la théorie des clusters. Et puis, faut-il rappeler l'indéniable attrait pour les ressources humaines créatives que présentent Cologne et, pardelà, la Rhénanie ?! C'est ce climat général d'effervescence créatrice qu'il s'agit de mettre à profit - comme d'autres régions en Europe misent sur l'attrait de leur climat plus ensoleillé.

Propos recueillis et traduits par I. Bourgeois

\section{Indications bibliographiques}

- BMWı, Gesamtwirtschaftliche Perspektiven der Kultur- und Kreativwirtschaft in Deutschland, Forschungsbericht $n^{\circ}$ 577, février 2009 (www.bmwi.de)

- Memi-InStiTUt, Mediencluster NRW: Innovation fördern, Strukturen bilden. Ausgangslage und Schwerpunkte für die Wirtschaftsförderung am Standort. Studie des memi-Institutes gefördert durch die Staatskanzlei NRW, Cologne, décembre 2007 (www.memi-koeln.de)

- Minister für Bundesangelegenheiten, EuRopa und Medien des Landes NRW, Medien.NRW. Gesucht: Die besten Ideen für Innovation, Konvergenz und Vernetzung im Medienbereich. Wettbewerbsaufruf und Leitfaden zum Förderwettbewerb, Düsseldorf, août 2008 (www.ziel2nrw.de, www.medien-nrw.de)

- www.creativeclass.com/richard_florida/. 